\title{
Application of DoE Methods to Establish a Model for the Pulsed Laser Deposition of PZT Thin-Films
}

\author{
A. Schatz and D. Pantel \\ Corporate Sector Research and Advance Engineering \\ Robert Bosch GmbH \\ Renningen, Germany \\ Andreas.Schatz@de.bosch.com
}

\author{
T. Hanemann \\ Department of Microsystems Engineering - IMTEK \\ University of Freiburg \\ Freiburg, Germany \\ Institute for Applied Materials - IAM \\ Karlsruhe Institute of Technology \\ Karlsruhe, Germany
}

\begin{abstract}
Controlling the growth of piezoelectric lead zirconate titanate $\left(\mathrm{Pb}\left[\mathrm{Zr}_{\mathrm{x}} \mathrm{Ti}_{1-\mathrm{x}}\right] \mathrm{O}_{3}-\mathrm{PZT}\right)$ thin-films is a key issue for their application in sensor and actuator devices. The pulsed laser deposition (PLD) process which is used in this work to grow PZT on platinized $150 \mathrm{~mm}$ silicon wafers can be easily modified by changing deposition parameters as the deposition pressure, deposition temperature, laser spot area on the target or laser pulse energy. In order to investigate the influence of these PLD parameters on the deposition rate and properties of the PZT thin-film as the leakage current density or the transverse piezoelectric coefficient $e_{31, f}$, statistical Design of Experiment methods were applied. Empirical models could be derived, describing the thin-film properties as function of the deposition parameters. It is observed that not only the PZT deposition parameters but also the parameters which are modified for the deposition of the $\mathrm{LaNiO}_{3}$ (LNO) template need to be controlled carefully. The parameters with the highest effect on the $e_{31, f}$ are shown to be the combination of deposition pressure and deposition temperature of the PZT layer, where either both high or low values lead to good PZT properties.
\end{abstract}

Keywords-PZT; MEMS; DoE; piezoelectric, model

\section{INTRODUCTION}

Piezoelectric thin-films made from $\mathrm{Pb}\left[\mathrm{Zr}_{\mathrm{x}} \mathrm{Ti}_{1-\mathrm{x}}\right] \mathrm{O}_{3}$ (PZT) have been under extensive research during the last decades and are attractive candidates for actuator and sensor applications within micro-electro-mechanical systems (MEMS) due to their outstanding piezoelectric properties [1]. Mass production tools for the deposition of PZT on wafer scale include chemical solution deposition and sputter deposition tools. Recently also the method of pulsed laser deposition (PLD) was made available for the deposition of PZT on large area substrates [2]. In general, the deposition parameters control the growth process of thin-films and hence the morphology. It is known that the structural properties of PZT thin-films influence the dielectric, ferroelectric and piezoelectric properties [3]. Controlling the growth of PZT hence is essential for successful implementation into MEMS devices. As the PLD itself is a versatile tool which allows easy modification of deposition parameters, e.g. substrate temperature, background gas and pressure or laser energy, the parameter range which can be used to grow PZT thin-films can be varied extensively. In this work we report on the application of statistical Design of Experiment (DoE) methods on the pulsed laser deposition of PZT thin-films for MEMS applications with two goals: first, the main influences of the PLD process on the deposition rate $R_{\text {dep }}$ and on PZT properties as the leakage current density $J_{\text {leak }}$ and the transverse piezoelectric coefficient $e_{31, f}$ are identified and second, empirical models for the PZT properties are derived. These models allow to predict the behavior of asgrown PZT thin-films and can be used for further process and film optimization.

\section{EXPERIMENTAL}

Silicon wafers of $150 \mathrm{~mm}$ diameter with a platinum bottom electrode (complete stack: Pt $(100 \mathrm{~nm}) / \mathrm{Ti}(10 \mathrm{~nm}) / \mathrm{SiO}_{2}$ $(2.4 \mu \mathrm{m}) / \mathrm{Si}$ (bulk)) were used as substrates. A PiezoFlare 800 PLD tool from Solmates B.V. was used to grow an approximately $10 \mathrm{~nm}$ thick buffer and template layer from $\mathrm{LaNiO}_{3}$ (LNO), the piezoelectric PZT thin-film in the range of $800 \mathrm{~nm}$ to $1 \mu \mathrm{m}$ thickness and a top LNO layer of approximately $40 \mathrm{~nm}$ thickness. The top LNO layer serves as additional oxidic electrode underneath the $100 \mathrm{~nm}$ thick platinum top electrode, which was sputtered and structured preceding the PLD step. The PLD process proceeds as follows: first, the substrate is heated by infrared heaters in base-pressure to the deposition temperature $T_{\text {dep }}$ (above the Curie temperature of PZT which is around $370{ }^{\circ} \mathrm{C}$ [4]), second, the oxygen flow is turned on and an oxygen background pressure $p_{\mathrm{O} 2}$ is established. All three PLD layers (LNO/PZT/LNO) are 
deposited without breaking the vacuum. The deposition settings for the first LNO layer and the PZT layer serve as factors for the experimental design and include the laser energy $E_{\text {laser }}$, the deposition temperature $T_{\text {dep }}$ and the oxygen pressure $p_{\mathrm{O} 2}$. An additional factor is the laser spot area $A_{\text {laserspot }}$ on the target which can be changed by implementing a metal mask in the beam path, cutting out an effective area of the laser pulse. $E_{\text {laser }}, p_{\mathrm{O} 2}$ and $A_{\text {laserspot }}$ were varied at two levels, $T_{\text {dep }}$ at three levels as shown in Tab. 1. The design space was spanned with the DoE software Cornerstone (camLine $\mathrm{GmbH}$ ) using a Doptimal design and setting 8 corners manually as inclusions, leading to a total of 40 runs (instead of 144 runs if a full factorial design was chosen). The deposition time was adapted individually (ranging from 60 to 180 minutes) to achieve a PZT thickness of approximately $1 \mu \mathrm{m}$ and after PLD deposition no further annealing step was applied.

The deposition rate $R_{\text {dep }}$ was calculated from the median thickness over the wafer radius (the PLD process is rotational symmetric) divided by the deposition time for the PZT layer. The thickness values were obtained from profilometer measurements after wet chemical etching of the LNO/PZT/LNO stack. For electrical measurement of the leakage current density $J_{\text {leak }}$, capacitors with $1 \mathrm{~mm}^{2}$ square area were used and a voltage ramp of $0.1 \mathrm{~V} / \mathrm{s}$ was applied (electric field from bottom to top electrode) by a sourcemeter (Keithley 2612A). The complete wafer was heated to $125^{\circ} \mathrm{C}$ during the measurement to avoid influences of ambient humidity. The leakage current density at an electric field of $100 \mathrm{kV} / \mathrm{cm}$ was calculated as the median of 10 measurements of different positions on every wafer. All wafers were diced in macroscopic cantilevers with $3.2 \mathrm{~mm} \cdot 25.2 \mathrm{~mm}$ area which were used in an $e_{31, f}$ measurement setup from aixACCT Systems GmbH. The $e_{31, f}$ values were obtained from the measurement of three cantilevers in converse mode and calculation of the derivative of the in-plane stress $\sigma_{1}$ versus the out-of-plane electric field $E_{3}$ $\left(e_{31, f}=-d \sigma_{1} / d E_{3}\right)$ according to Mazzalai et al. [5].

TABLE I. DEPOSITION PARAMETERS AND ASSOCIATED LOW, (CENTER) AND HIGH-VALUES USED FOR THE EXPERIMENTAL DESIGN

\begin{tabular}{|l|l|l|l|}
\hline \multicolumn{1}{|c|}{ Deposition parameter } & \multicolumn{1}{|c|}{ Low } & Center & \multicolumn{1}{c|}{ High } \\
\hline$E_{\text {laser,LNO }}$ and $E_{\text {laser,PZT }}$ & $480 \mathrm{~mJ} /$ pulse & - & $660 \mathrm{~mJ} /$ pulse \\
\hline$p_{\mathrm{O} 2, \mathrm{LNO}}$ and $p_{\mathrm{O} 2, \mathrm{PZT}}$ & $0.05 \mathrm{mbar}$ & - & $0.20 \mathrm{mbar}$ \\
\hline$T_{\text {dep,LNO }}$ and $T_{\text {dep,PZT }}$ & $445^{\circ} \mathrm{C}$ & $510^{\circ} \mathrm{C}$ & $570{ }^{\circ} \mathrm{C}$ \\
\hline$A_{\text {laserspot }}$ & $2.56 \mathrm{~mm}^{2}$ & - & $4.00 \mathrm{~mm}^{2}$ \\
\hline
\end{tabular}

\section{RESULTS AND DISCUSSION}

Linear interaction of the seven DoE factors results together with two quadratic terms $\left(T_{\mathrm{dep}, \mathrm{LNO}}{ }^{2}\right.$ and $\left.T_{\mathrm{dep}, \mathrm{PZT}^{2}}{ }^{2}\right)$ in a total of 30 terms which serve as coefficients in a multiple linear regression. The laser energy $E_{\text {laser,LNO/PZT was logged during the }}$ PLD process internally within the excimer laser and additionally by an external laser power meter which is placed after a beam splitter in the beam path (preceding the metal mask which truncates the laser pulse area-wise). As the external power meter data was fluctuating and the laser energy logging could not catch these fluctuations, we expect the external power meter data to be more precise for describing the influence of the effective laser energy. Hence we replaced the laser energy factors $E_{\text {laser,LNO/PZT }}$ with the laser power factors $P_{\text {laser,LNO/PZT }}$ which are combinatory factors of the laser energy $E_{\text {laser,LNO/PZT }}$ and the laser spot area $A_{\text {laserspot }}$.

After implementing all data (factors and responses) into the DoE software, a stepwise backward regression was performed for each of the responses removing the least significant terms if the p-value exceeds 0.1 . This results in a total of 8,18 and 11 terms for the responses $R_{\text {dep }}, e_{31, f}$ and $J_{\text {leak }}$, respectively. In order to achieve a high adjusted coefficient of determination $R^{2}$ adj $\left(R^{2}\right.$ is not used due to low population size), the response variable of $J_{\text {leak }}$ was transformed by taking the square root $\left(J_{\text {leak }}^{1 / 2}\right)$. The $R_{\text {adj }}^{2}$ of the linear regressions of $R_{\text {dep }}, e_{31, f}$ and $J_{\text {leak }}$ were calculated to be $0.98,0.92$ and 0.90 , respectively. The residuals of each regression show now trend in time (hence they are homoscedastic) and are normally distributed (identified from residuals-probability plots) which allows application of the regression models and serves as validation of the models.

The terms of the regressions with the highest effects can be assessed from the Pareto charts of effects (see Fig. 1 as example for the $R_{\text {dep }}$ ) to be the laser power for the PZT layer $P_{\text {laser,PZT }}$ (mainly effecting $R_{\text {dep }}$ and $e_{31, f}$ ), the oxygen pressure during the PZT deposition $p_{\mathrm{O} 2, \mathrm{PZT}}$ (mainly effecting $R_{\text {dep }}$ and $\left.J_{\text {leak}}\right)$ and the combination of deposition temperature and pressuring during PZT growth $T_{\text {dep,PZT }} \cdot p_{\mathrm{O} 2 \text {,PZT }}$ (mainly effecting $e_{31, f}$ and $\left.J_{\text {leak }}\right)$. The terms containing LNO deposition parameters mathematically show less effect.

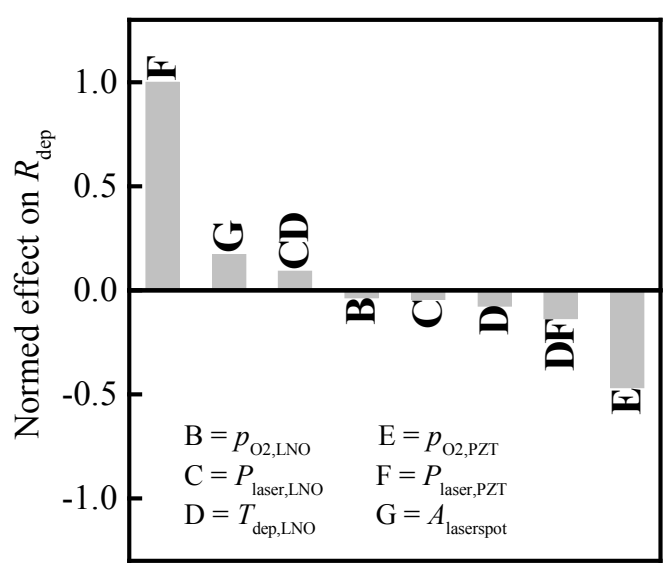

Fig. 1. Pareto chart of effects for the deposition rate $R_{\text {dep }}$ (normed on the highest effect). 


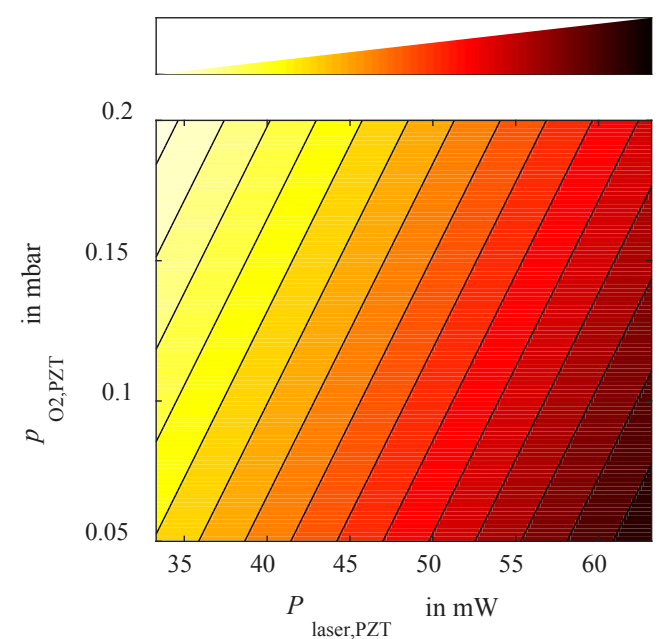

Fig. 2. Contour plot of the deposition rate $R_{\text {dep }}$ with variable $P_{\text {laser,PZT }}$ and $p_{\mathrm{O} 2, \mathrm{PZT}}$, maintaining the other factors constant.

The empirical models (i.e. the regression equations) for the three responses are used to create contour plots where the response value is color coded and the $\mathrm{x}$ - and $\mathrm{y}$-axis correspond to the main effecting terms. The other factors were kept constant at the center point of the DoE data range $\left(T_{\text {dep,LNO }}=\right.$ $510{ }^{\circ} \mathrm{C}, p_{\mathrm{O} 2, \mathrm{LNO}}=0.125 \mathrm{mbar}, P_{\text {laser }, \mathrm{LNO}}=13.3 \mathrm{~mW}, P_{\text {laser, } \mathrm{PZT}}=$ $39.4 \mathrm{~mW}, A_{\text {laserspot }}=2.56 \mathrm{~mm}^{2}$ ). From the contour plot in Fig. 2 , a linear dependence of $R_{\mathrm{dep}}$ on its main effecting terms $P_{\text {laser,PZT }}$ and $p_{\mathrm{O} 2 \mathrm{PZT}}$ can be observed: higher laser power or lower deposition pressure leading to a higher deposition rate. The models of the transverse piezoelectric coefficient and the leakage current density are depicted in Fig. 3 and Fig. 4, respectively, with $T_{\text {dep,PZT }}$ serving as x-axis and $p_{\mathrm{O} \text {,PZT }}$ as $\mathrm{y}$ -

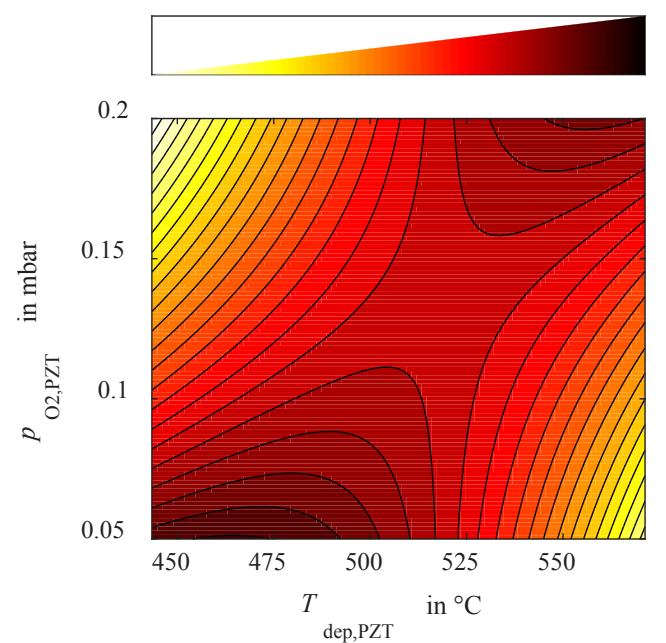

Fig. 3. Contour plot of the model response of the transverse piezoelectric coefficient $e_{31, f}$ with variable $T_{\mathrm{dep}, \mathrm{PZT}}$ and $p_{\mathrm{O} 2, \mathrm{PZT}}$, maintaining all other factors constant. axis. The shape of both plots looks rather similar if the $J_{\text {leak }}$ plot can be seen as being shifted to higher $T_{\text {dep,PZT }}$ values with an offset of approximately $30{ }^{\circ} \mathrm{C}$ and being stretched along the $\mathrm{y}$ axis $\left(p_{\mathrm{O}, \mathrm{PZT}}\right)$. Generally, a major trend can be derived from both plots regarding PZT thin-films with good piezoelectric and electric properties: low $J_{\text {leak }}$ and high $\left|e_{31, f}\right|$ values can be reached for either both low or both high deposition pressure $p_{\mathrm{O} 2 \text {, PZT }}$ and temperature $T_{\mathrm{dep}, \mathrm{PZT}}$. For further process optimization of the $e_{31, f}$, no special care has to be taken regarding the leakage current density if the $T_{\mathrm{dep}, \mathrm{PZT}}$ and $p_{\mathrm{O} 2, \mathrm{PZT}}$ are changed accordingly.

From literature it is known that the template for the growth of PZT itself is of importance regarding the PZT properties (the PZT orientation and the grain size is dependent on the template and hence the dielectric permittivity [6] and the leakage current density is dependent on the oxygen partial pressure during LNO growth via sputtering [7]). For this reason, the PLD deposition parameters for the LNO template used were included in the DoE. As the terms with the highest effect on the $e_{31, f}$ are identified as terms which include PZT deposition parameters and the terms which include LNO deposition parameters are only of minor effect, it is investigated whether the LNO deposition parameters need to be considered (both, in reality and in the model). A second model was created, excluding all terms containing LNO-related factors. The measured values of the $e_{31, f}$ of three samples (\#1-\#3) were compared with calculated values from both the full model (including LNO deposition terms) and the reduced model (excluding LNO deposition terms) and are displayed in Fig. 5. The error bars represent the measurement error for the fabricated sample values and the $90 \%$ confidence interval of both models.

Though all error bars are overlapping, the model without LNO-terms is not exact enough as it is not covering the decreasing trend of the $\left|e_{31, f}\right|$ with increasing $T_{\text {dep,PZT }}$ (see Fig. 5 and compare Fig. 3 at $p_{\mathrm{O} 2, \mathrm{PZT}}=0.05$ mbar). It can be concluded

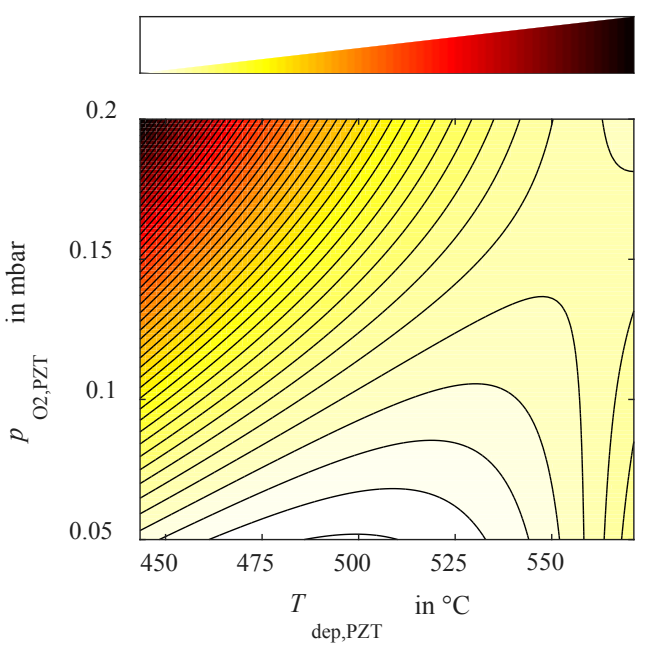

Fig. 4. Contour plot of the model response of the leakage current density $J_{\text {leak }}$ (at $E=100 \mathrm{kV} / \mathrm{cm}$ when a voltage ramp of $0.1 \mathrm{~V} / \mathrm{s}$ is applied) with variable $T_{\text {dep,PZT }}$ and $p_{\mathrm{O} 2, \mathrm{PZT}}$, maintaining all other factors constant. 


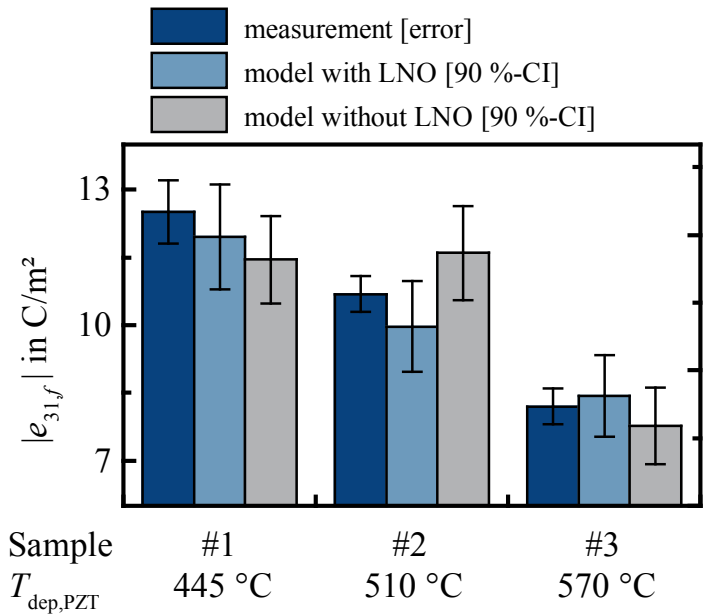

Fig. 5. Comparison of the $\left|e_{31, f}\right|$ value for three samples deposited at $p_{\mathrm{O} 2 \text {, PZT }}=0.05$ mbar and varying other factors (the value of $T_{\text {dep,PZT }}$ is shown in the graph as it has the highest effect), derived from real measurement (dark blue), full model (light blue) and model without LNO terms (grey).

that the LNO growth parameters indeed play a role regarding PZT thin-film properties as shown for the $e_{31, f}$.

\section{CONCLUSION}

To investigate the influence of the deposition parameters (substrate temperature, oxygen pressure, laser pulse energy and laser spot area on the target) of a PLD tool on the deposition rate, the transverse piezoelectric coefficient and the leakage current density of PZT thin-films, statistical Design of Experiment methods were applied. Main influences on the deposition rate turns out to be the laser power (a combinatory factor of the laser pulse energy and the laser spot area) and the deposition pressure. The main effect on the $e_{31, f}$ and the leakage current density was identified to be the combination of deposition pressure and temperature with both parameters either high or low leading to good piezoelectric and electrical properties. The influences of the deposition parameters of the LNO layer, which is used as a template for the PZT growth, were also investigated within the DoE and it was concluded that though their effect is not too high, the LNO deposition parameters must not be neglected when modelling the $e_{31, f}$ and hence they are to be controlled when fabricating PZT thinfilms.

\section{ACKNOWLEDGMENT}

The authors would like to thank Solmates B.V. for process support on the PLD tool.

\section{REFERENCES}

[1] S. Trolier-McKinstry and P. Muralt, "Thin film piezoelectrics for MEMS," J. Electroceram., vol. 12, pp. 7-17, 2004

[2] J.A. Greer, "History and current status of commercial pulsed laser deposition equipment," J. Phys. D: Appl. Phys., vol. 47, p. 034005, 2014

[3] W.C. Goh, K. Yao, and C.K. Ong, "Effects of microstructure on the properties of ferroelectric lead zirconate titanate (PZT) thin films," Appl. Phys. A, vol. 81, pp. 1089-1093, 2005

[4] B. Jaffe, W.R. Cook, and H. Jaffe, "Piezoelectric Ceramics," Academic Press, New York, 1971

[5] A. Mazzalai, D. Balma, N. Chidambaram, R. Matloub, and P. Muralt, "Characterization and fatigue of the converse piezoelectric effect in PZT films for MEMS applications," J. MEMS, vol. 24, pp. 831-838, 2015

[6] N. Sama et al., "On the influence of the top and bottom electrodes - A comparative study between Pt and LNO electrodes for PZT thin films," J. Cryst. Growth, vol. 310, pp. 3299-3302, 2008

[7] H. Kim, J.-H. Kim, and W. Choo, "Ferroelectric properties of PZT films on LaNiO3 bottom electrode deposited under different oxygen partial pressure," J. Eur. Ceram. Soc., vol. 25, pp. 2253-2256, 2005 